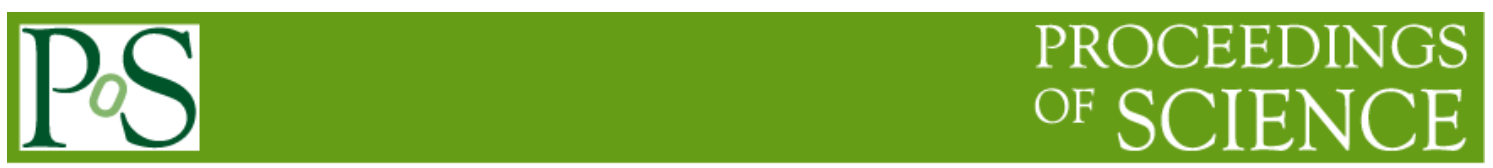

\title{
Searches for gravitational effects at the TeV scale with the ATLAS detector
}

\author{
Tracey Berry ${ }^{1}$ on behalf of the ATLAS Collaboration \\ Royal Holloway, University of London \\ E-mail: tracey.berry@rhul.ac.uk
}

The Standard Model presently does not explain why the gravitational force is weaker than the other forces. New physics models, for example those postulating the existence of extra dimensions, have been proposed to address this. This paper describes searches for gravitational effects performed by the ATLAS collaboration using data from proton-proton collisions recorded in 2011 at $V_{\mathrm{s}}=7 \mathrm{TeV}$ and in 2012 at $V_{\mathrm{s}}=8 \mathrm{TeV}$, provided by the Large Hadron Collider (LHC).

The European Physical Society Conference on High Energy Physics 18-24 July, 2013

Stockholm, Sweden

1

Speaker 


\section{Introduction}

Since the recent discovery of a Higgs-like boson which has a relatively low mass, there is even more motivation to search for physics beyond the Standard Model (SM). Fundamental questions remain unanswered, for example why is gravity so weak as compared with the other forces and why is the Planck scale $\left(10^{19} \mathrm{GeV}\right)$ so much larger than the electroweak scale $\left(10^{3}\right.$ $\mathrm{GeV}$ ), referred to as the hierarchy problem. In attempts to answer these, several extra dimensional models have been introduced [1,2]. This paper describes the searches for gravitational effects peformed by the ATLAS Collaboration. These are divided into three sections: searches for evidence of large extra dimensions, black holes and warped extra dimensions.

The ATLAS detector is a multi-purpose general detector [3], situated at the Large Hadron Collider (LHC), at the CERN laboratory in Geneva, Switzerland. In 2011 ATLAS collected proton-proton collision data at a centre of mass energy of $7 \mathrm{TeV}$, and in 2012 this increased to $8 \mathrm{TeV}$. The detector is composed of subsystems designed to measure the energy and momentum of photons, electrons, muons, jets and detect the existence of missing energy up to a few $\mathrm{TeV}$, which enables searches for gravitational effects to be performed.

\section{Large Extra Dimension Searches}

The Arkani-Hamed, Dimopoulos, Dvali (ADD) model addresses the apparent weakness of the gravitational strength and the hierarchy problem by introducing many large extra dimensions (LED), in which the gravitational field can be thought of as being diluted, as it is spread out throughout the large extra dimensions [1].

The ADD model parameters are the number of extra dimensions (n) and the effective Planck mass in the $4+n$ dimensions $\left(M_{D}\right)$, which can be related to the Planck scale $\left(M_{P l}\right)$ (via Gauss's law) and the size of the extra dimensions (R) as described in Equation 1 [1].

$$
\text { Equation 1: } \quad \mathrm{M}_{\mathrm{Pl}}{ }^{2}=\mathrm{M}_{\mathrm{D}}{ }^{(2+\mathrm{n})} \mathrm{R}^{\mathrm{n}} \text {. }
$$

ATLAS presently searches for these types of models by searching for excesses in the numbers of events with the signature of jet(s) plus missing energy and photon(s) plus missing energy caused by graviton emission; for non-resonant deviations in the dilepton, diphoton or dijet spectrum caused by gravitron exchange; and production of black holes.

\subsection{Graviton Emission: Monojet \& Monophoton Searches}

The strongest graviton emission limits set by ATLAS are presently obtained from the monojet search [4]. This search was performed using $10.5 \mathrm{fb}^{-1}$ of $8 \mathrm{TeV}$ data, collected in the 2012 LHC run. Several signal regions were used in the ATLAS analysis, the one which provided the best expected limits was one which searched for excesses in the number of events which contained both missing transverse energy $\left(\mathrm{E}_{\mathrm{T}}{ }^{\text {miss }}\right.$ ) and a leading jet with transverse momentum $\left(\mathrm{p}_{\mathrm{t}}\right)$ in the central part of the detector (with pseudorapidity less than 2) greater than $350 \mathrm{GeV}$. Additionally, events were selected such that they contained at most two jets with $\mathrm{p}_{\mathrm{t}}>$ $120 \mathrm{GeV}$ (with pseudorapidity less than 4.5) and the difference in the angle $(\phi)$ between the 
second-leading jet and the missing energy was required to be greater than 0.5 . The dominant background in this case comes from $\mathrm{Z}$ bosons decaying to 2 neutrinos plus jets and $\mathrm{W}$ bosons decaying to a lepton and a neutrino plus jets. In this search region a total background of $2180 \pm$ 70 (statistical uncertainty on the electro-weak data-driven background estimation) \pm 120 (statistical uncertainty of the Monte Carlo) \pm 100 (systematic uncertainty) is expected and 2353 were observed. The lower mass limits on $\mathrm{M}_{\mathrm{D}}$ obtained, at the $95 \%$ confidence level (CL), range from $2.7 \mathrm{TeV}(\mathrm{n}=2)$ to $4.3 \mathrm{TeV}(\mathrm{n}=6)$. The expected and observed $\mathrm{E}_{\mathrm{T}}{ }^{\text {miss }}$ distribution and the limits determined are displayed on the left and right of Figure 1 respectively.
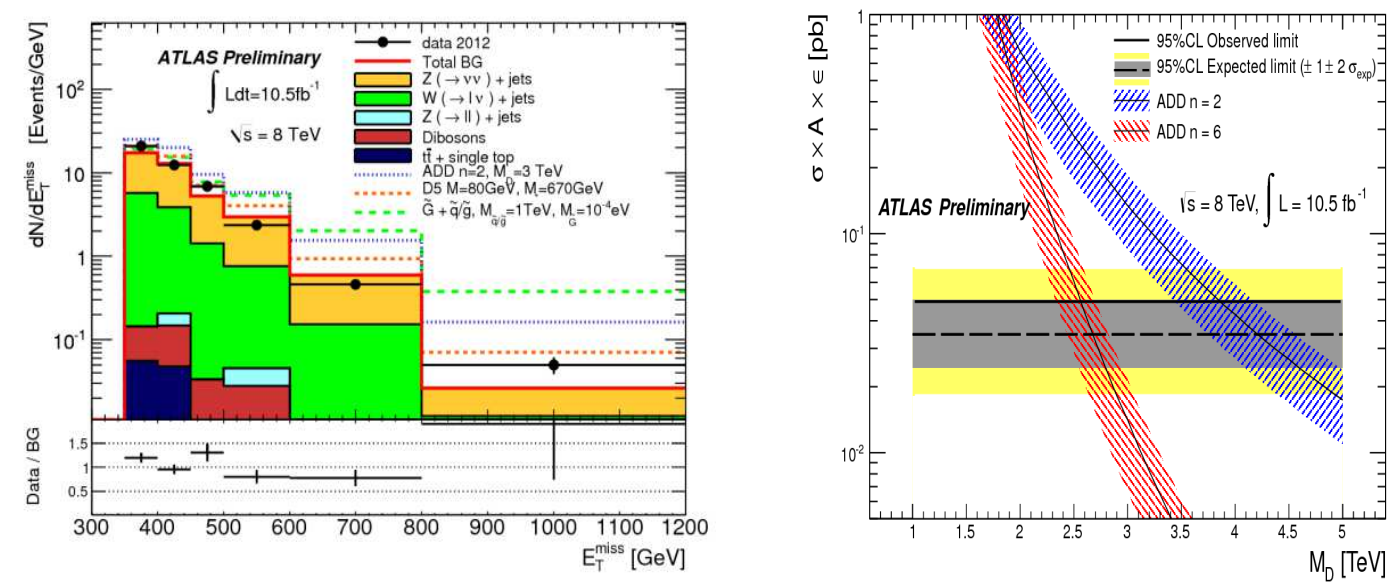

Figure 1: Left: Measured distributions (black dots) in signal region compared to the predictions for SM backgrounds (histograms). Only statistical uncertainties are shown. For illustration purposes, the impact of different ADD are included. Right: The predicted ADD cross-section $\times$ acceptance $\times$ effciency $(\sigma \times \mathrm{A}$ $\times \varepsilon$ ) for the selection as a function of $M_{D}$ for $n=2$ and $n=6$, where bands represent the uncertainty on the theory. For comparison, the model-independent observed (solid line) and expected (dashed line) $95 \% \mathrm{CL}$ limits on $\sigma \times \mathrm{A} \times \varepsilon$ are shown. The shaded areas around the expected limit indicate the expected $\pm 1 \sigma$ and $\pm 2 \sigma$ ranges of limits in the absence of a signal [4].

These jet plus missing energy limits exceed the limits obtained in a search using $4.6 \mathrm{fb}^{-1}$ of data selected to contain a photon and missing energy collected at $\sqrt{s}_{\mathrm{s}}=7 \mathrm{TeV}$ [5]. In which excluded mass limits on $\mathrm{M}_{\mathrm{D}}$ obtained improved those previously set from the LEP and Tevatron experiments and ranged from $1.93(n=2)$ to $1.89(n=6)$ : displayed on the right of Figure 2 [5].

\subsection{Graviton Exchange: Dilepton and Diphoton Searches}

An alternative way to search for the ADD LED model is via graviton exchange rather than graviton emission. In this case, limits are set on the scale for the onset of quantum gravity $\left(\mathrm{M}_{\mathrm{s}}\right)$, which is related to $M_{D}$ [6]. Experimentally, graviton exchange could lead to deviations in the invariant mass continuum spectrum of dilepton, diphoton or dijet data. ATLAS has searched in the dilepton channel using $5 \mathrm{fb}^{-1}$ of the $7 \mathrm{TeV}$ data and combined this with a diphoton search, using the same dataset [6][7]. The dilepton search was performed selecting data containing 2 high $\mathrm{p}_{\mathrm{t}}$ opposite sign muons or 2 high $\mathrm{p}_{\mathrm{t}}$ electrons and using an optimized search region 
requiring the dilepton pair invariant mass $\left(\mathrm{m}_{\mathrm{Il}}\right)$ to be above $1300 \mathrm{GeV}$. The dominant background is from the irreducible Drell-Yan contribution. The expected and observed dielectron $\left(\mathrm{m}_{\mathrm{ee}}\right)$ invariant mass distributions is shown on the right of Figure 2. The limits obtained were combined (removing any overlap with the electrons) with a search performed using ATLAS diphoton data [7]. The combined lower mass exclusion limit on $\mathrm{M}_{\mathrm{s}}$ ranged from $2.95 \mathrm{TeV}(\mathrm{n}=6)$ to $4.18(\mathrm{n}=3)$ in the HLZ [8] naming convention; was $3.14 \mathrm{TeV}$ in the Hewitt [9] and $3.51 \mathrm{TeV}$ in the GRW [10] convention for results determined using a prior flat in $1 / \mathrm{M}_{\mathrm{S}}{ }^{4}$ [6].
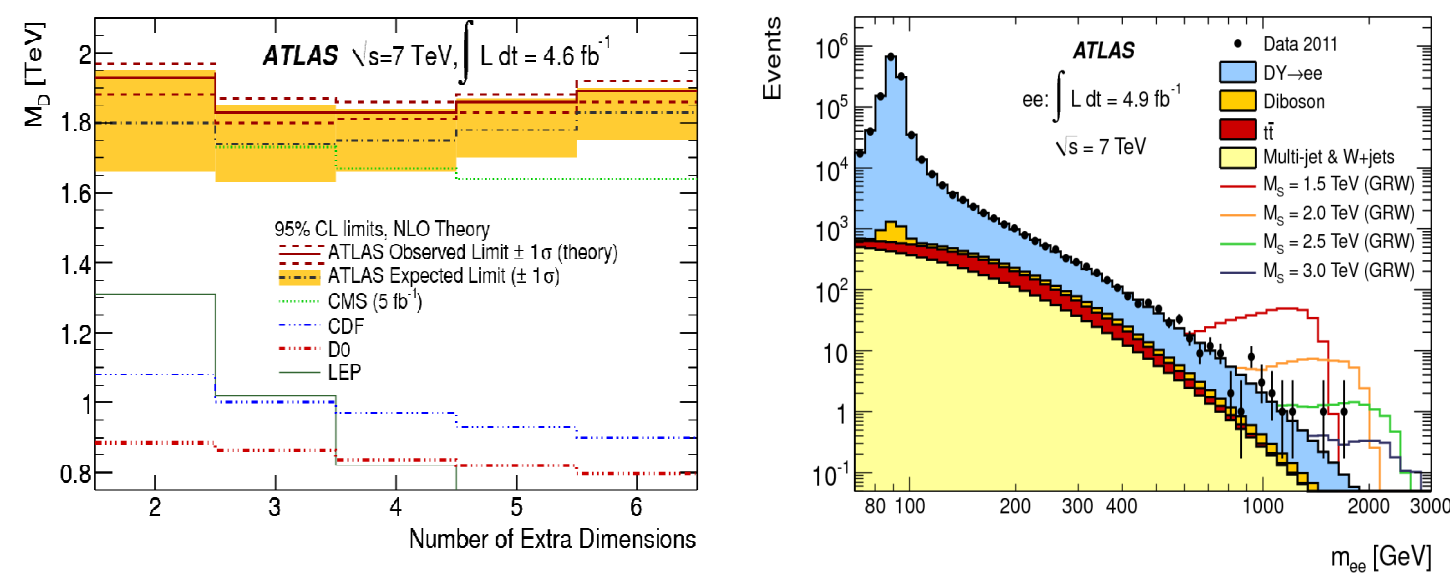

Figure 2: Left: Observed (solid lines) and expected (dashed-dotted lines) 95\% CL limits on $\mathrm{M}_{\mathrm{D}}$ as a function of the number of extra spatial dimensions $n$ in the ADD LED model [5]. Right: Reconstructed dielectron invariant mass distribution for data and Monte Carlo backgrounds. Also shown are the predictions for different ADD model $\mathrm{M}_{\mathrm{S}}$ values. The bin width is constant in $\log \left(\mathrm{m}_{\mathrm{ll}}\right)$ [6].

\section{Black Hole Searches}

If gravity becomes strong at the $\mathrm{TeV}$ scale, it could be strong enough to produce microscopic black holes which would decay through Hawking radiation. These are detectable in the ATLAS detector via decays which are characteristically democratic and isotropic, therefore searches are performed for final states containing (many) jets (and leptons) at high mass.

ATLAS has performed searches in several different channels using $7 \mathrm{TeV}$ data: multijet [11], lepton+jets [12] and same-sign dimuon [13] (which was updated to use $8 \mathrm{TeV}$ data [14]) and the $8 \mathrm{TeV}$ result described below.

\subsection{Non-Thermal Quantum Black Holes: Photon and Jet Search}

ATLAS searched for evidence of non-thermal quantum black holes (QBH), using the full dataset collected in 2012, $20.3 \mathrm{fb}^{-1}$. This was performed by studying the $(\gamma+\mathrm{jet})$ mass distribution and comparing it to a background model fit from data [15]. The signature of a QBH would be a very striking excess of events at high mass, as demonstrated on the left of Figure 3. This is compared to a background model fit from data. ATLAS set $95 \%$ confidence limits on 
non-thermal QBH, excluding masses below 4.6 TeV and also set limits on generic Gaussianshaped signals.

\section{Warped Extra Dimensional Searches}

Another model which addresses the hierarchy problem is the Randall-Sundrum 1 model [2]. In this model one extra dimension is introduced in which only gravity propagates. The Planck scale is reduced to the electroweak scale by an exponential warping of the additional spatial dimension. This model introduces the possibility of graviton exchange. The model can be parameterised in terms of the mass of the lightest excitation $\left(\mathrm{m}_{\mathrm{G}}\right)$ and the coupling $\mathrm{k} / \mathrm{M}_{\mathrm{Pl}}$, where $\mathrm{k}$ is a measure of the curvature of the additional special dimension. The experimental signature for this model would be a resonance (or series of resonances) at high mass in the dielectron, dimuon, ditau, dijet or diboson invariant mass spectra. ATLAS has performed searches for evidence of Randall-Sundrum model gravitons in several channels: dilepton, diphoton and dijet. The most stringent ATLAS limits presently come from dilepton channel searches using the full 2012 dataset of $20 \mathrm{fb}^{-1}$ [16]. ATLAS performs the search for 2 high $\mathrm{p}_{\mathrm{t}}$ electrons or opposite-sign muons. Backgrounds are normalised to the data in the Z-peak region. Figure 3 (right) shows the expected and observed dielectron invariant mass spectra. Templates are fitted to obtain a lower mass limit for the graviton of $2.47 \mathrm{TeV}$ for a coupling of $\mathrm{k} / \mathrm{M}_{\mathrm{Pl}}=0.1$.
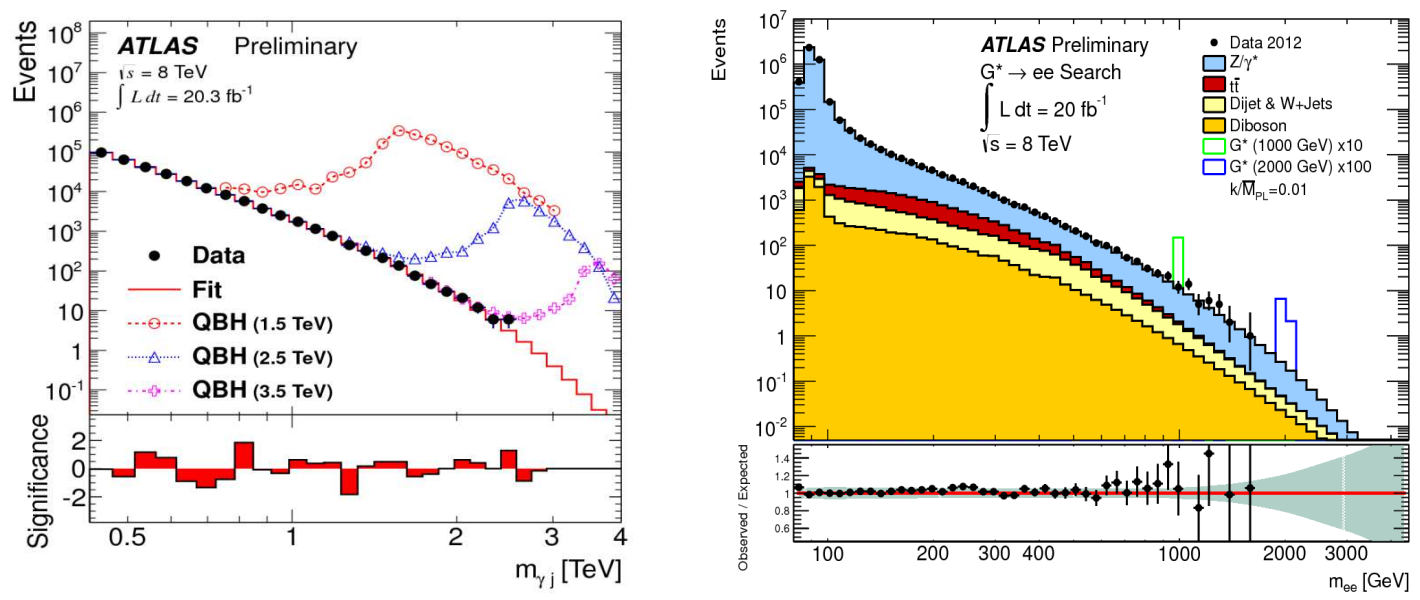

Figure 3: Left: Invariant mass of the $\gamma+$ jet pair $\left(\mathrm{m}_{\gamma \mathrm{j}}\right)$ for events passing the final selections. The bin widths are chosen to be twice the mass resolution at the centre of each bin. Overlaid is the fitted background function integrated over each bin (solid line), with three examples of QBH signals. For better visibility the QBH signals are only drawn for $\mathrm{m}_{\gamma \mathrm{j}}$ starting at half the threshold mass, and for the $1.5 \mathrm{TeV}$ signal it ends at twice the threshold mass. Lower panel: The statistical significance of the difference between data and background in each bin [15]. Right: Dielectron invariant mass $\left(\mathrm{m}_{\mathrm{ee}}\right)$ distribution after dielectron selection with statistical uncertainties after final selection, compared to the stacked sum of all expected backgrounds, with two selected graviton $\left(\mathrm{G}^{*}\right)$ signals overlaid. The bin width is constant in log $\mathrm{m}_{\mathrm{ee}}$. Lower: The points show the ratio of observed to expected events with statistical uncertainty, while the shaded band indicates the mass-dependent systematic uncertainty on the sum of the backgrounds [16]. 


\section{Conclusion and Outlook}

No evidence for gravitational effects has yet been observed by the ATLAS collaboration. Searches continue using the full $8 \mathrm{TeV}$ dataset, and are proposed in the future using the higher centre of mass energy and luminosity which is anticipated in the future at the LHC.

\section{References}

[1] N. Arkani-Hamed, S. Dimopoulos, and G. Dvali, Phys. Lett. B 429, 263 (1998).

[2] L. Randall and R. Sundrum, Phys. Rev. Lett. 83, 3370 (1999) .

[3] ATLAS Collaboration, JINST 3, S08003 (2008).

[4] ATLAS Collaboration, Search for new phenomena in monojet plus missing transverse momentum final states using 10/fb of pp collisions at $\sqrt{ } s=8 \mathrm{TeV}$ with the ATLAS detector at the LHC, ATLAS-CONF-2012-147. https://atlas.web.cern.ch/Atlas/GROUPS/PHYSICS/CONFNOTES/ATLAS-CONF-2012-147/

[5] ATLAS Collaboration, Search for dark matter candidates and large extra dimensions in events with a photon and missing transverse momentum in pp collision data at $\sqrt{ } s=7$ TeV with the ATLAS detector, Phys. Rev. Lett. 110, 011802 (2013). https://atlas.web.cern.ch/Atlas/GROUPS/PHYSICS/PAPERS/EXOT-2011-20/

[6] ATLAS Collaboration, Search for contact interactions and large extra dimensions in dilepton events from pp collisions at $\sqrt{s}_{s}=7$ TeV with the ATLAS detector, Phys. Rev. D 87, 015010 (2013). https://atlas.web.cern.ch/Atlas/GROUPS/PHYSICS/PAPERS/EXOT-2012-17/

[7] ATLAS Collaboration, Search for extra dimensions in diphoton events using proton-proton collisions recorded at $\sqrt{ }_{s}=7 \mathrm{TeV}$ with the ATLAS detector at the LHC, Nucl. Jour. of Phys. 15, 043007 (2013). https://atlas.web.cern.ch/Atlas/GROUPS/PHYSICS/PAPERS/EXOT-2012-06/

[8] T. Han, J. D. Lykken, and R.-J. Zhang, Phys. Rev. D 59, 105006 (1999).

[9] J. L. Hewett, Phys. Rev. Lett. 82, 4765 (1999).

[10] G. F. Giudice, R. Rattazzi, and J. D. Wells, Nucl. Phys. B 544, 3 (1999).

[11] ATLAS Collaboration, Search for microscopic black holes in multi-jet final states with the ATLAS detector at $\sqrt{ }_{s}=7 \mathrm{TeV}$, ATLAS-CONF-2011-068.

[12] ATLAS Collaboration, Search for TeV-scale gravity signatures in final states with leptons and jets with the ATLAS detector at $\sqrt{ } s=7 \mathrm{TeV}$, Phys. Lett. B 716 (2012) 122.

[13] ATLAS Collaboration, Search for strong gravity signatures in same-sign dimuon final states using the ATLAS detector at the LHC, Phys. Lett. B709 (2012) 322.

[14] ATLAS Collaboration, Search for microscopic black holes in a like-sign final dimuon final state using large track multiplicity with the ATLAS detector at the LHC, Phys. Rev. D88, 072001 (2013).

[15] ATLAS Collaboration, Search for new phenomena in photon-jet events collected in protonproton collisions at $\sqrt{s}_{s}=8 \mathrm{TeV}$ with the ATLAS detector, arXiv:1309.3230. https://atlas.web.cern.ch/Atlas/GROUPS/PHYSICS/PAPERS/EXOT-2012-22/

[16] ATLAS Collaboration, Search for high-mass dilepton resonances in 20/fb of pp collisions at $\sqrt{s}_{s}$ $=8 \mathrm{TeV}$ with the ATLAS experiment, ATLAS-CONF-2013-017.

https://atlas.web.cern.ch/Atlas/GROUPS/PHYSICS/CONFNOTES/ATLAS-CONF-2013-017/ 